\title{
THE LEAST EIGENVALUE OF GRAPHS WHOSE COMPLEMENTS ARE UNICYCLIC
}

\author{
Yi WANG ${ }^{1}$, Yi-Zheng FAN ${ }^{1}$, XIAO-XIN LI ${ }^{2}$ \\ AND \\ FEI-FEI ZHANG ${ }^{1}$ \\ ${ }^{1}$ School of Mathematical Sciences \\ Anhui University, Hefei 230601, P.R. China \\ 2 Department of Mathematics and Computer Sciences \\ Chizhou University, Chizhou 247000, P.R. China \\ e-mail: wangy@ahu.edu.cn \\ fanyz@ahu.edu.cn \\ lxx@czu.edu.cn \\ zhangfeifei2403@126.com
}

\begin{abstract}
A graph in a certain graph class is called minimizing if the least eigenvalue of its adjacency matrix attains the minimum among all graphs in that class. Bell et al. have identified a subclass within the connected graphs of order $n$ and size $m$ in which minimizing graphs belong (the complements of such graphs are either disconnected or contain a clique of size $\frac{n}{2}$ ). In this paper we discuss the minimizing graphs of a special class of graphs of order $n$ whose complements are connected and contains exactly one cycle (namely the class $\mathscr{U}_{n}^{c}$ of graphs whose complements are unicyclic), and characterize the unique minimizing graph in $\mathscr{U}_{n}^{c}$ when $n \geq 20$.
\end{abstract}

Keywords: unicyclic graph, complement, adjacency matrix, least eigenvalue.

2010 Mathematics Subject Classification: 05C50, 05D05, 15 A18.

\section{REFERENCES}

[1] F.K. Bell, D. Cvetković, P. Rowlinson and S. Simić, Graph for which the least eigenvalues is minimal, I, Linear Algebra Appl. 429 (2008) 234-241. doi:10.1016/j.laa.2008.02.032 
[2] F.K. Bell, D. Cvetković, P. Rowlinson and S. Simić, Graph for which the least eigenvalues is minimal, II, Linear Algebra Appl. 429 (2008) 2168-2179. doi:10.1016/j.laa.2008.06.018

[3] D. Cvetković and P. Rowlinson, The largest eigenvalues of a graph: a survey, Linear Multilinear Algebra 28 (1990) 3-33. doi:10.1080/03081089008818026

[4] D. Cvetković, P. Rowlinson and S. Simić, Spectral Generalizations of Line Graphs: on Graph with Least Eigenvalue -2 (London Math. Soc., LNS 314, Cambridge Univ. Press, 2004).

[5] Y.-Z. Fan, Y. Wang and Y.-B. Gao, Minimizing the least eigenvalues of unicyclic graphs with application to spectral spread, Linear Algebra Appl. 429 (2008) 577-588. doi:10.1016/j.laa.2008.03.012

[6] Y.-Z. Fan, F.-F Zhang and Y. Wang, The least eigenvalue of the complements of trees, Linear Algebra Appl. 435 (2011) 2150-2155. doi:10.1016/j.laa.2011.04.011

[7] Y. Hong and J. Shu, Sharp lower bounds of the least eigenvalue of planar graphs, Linear Algebra Appl. 296 (1999) 227-232. doi:10.1016/S0024-3795(99)00129-9

[8] R. Liu, M. Zhai and J. Shu, The least eigenvalues of unicyclic graphs with $n$ vertices and $k$ pendant vertices, Linear Algebra Appl. 431 (2009) 657-665. doi:10.1016/j.laa.2009.03.016

[9] M. Petrović, B. Borovićanin and T. Aleksić, Bicyclic graphs for which the least eigenvalue is minimum, Linear Algebra Appl. 430 (2009) 1328-1335. doi:10.1016/j.laa.2008.10.026

[10] M. Petrović, T. Aleksić and S. Simić, Further results on the least eigenvalue of connected graphs, Linear Algebra Appl. 435 (2011) 2303-2313. doi:10.1016/j.laa.2011.04.030

[11] Y.-Y. Tan and Y.-Z. Fan, The vertex (edge) independence number, vertex (edge) cover number and the least eigenvalue of a graph, Linear Algebra Appl. 433 (2010) 790-795. doi:10.1016/j.laa.2010.04.009

[12] Y. Wang, Y. Qiao and Y.-Z. Fan, On the least eigenvalue of graphs with cut vertices, J. Math. Res. Exposition 30 (2010) 951-956.

[13] Y. Wang and Y.-Z. Fan, The least eigenvalue of a graph with cut vertices, Linear Algebra Appl. 433 (2010) 19-27. doi:10.1016/j.laa.2010.01.030

[14] M.-L. Ye, Y.-Z. Fan and D. Liang, The least eigenvalue of graphs with given connectivity, Linear Algebra Appl. 430 (2009) 1375-1379. doi:10.1016/j.laa.2008.10.031 